all restricted and for which central approval is automatic.

Applications for new consultant posts are initiated at District level and a useful guide as to the possible success of an application would be the position of the District in relation to the national average of psychiatrists per unit of population. At present there is roughly one psychiatrist in mental illness for 50,000; one child and adolescent psychiatrist for 150,000; and one in mental handicap for 200,000 . It is not likely that sufficiently radical changes will have occurred in the infrastructure to provide very different numbers for expansion in 1983-84, and this makes it likely that roughly the same numbers of new posts could be created as in the previous year-up to 35 in adult mental illness; 15 in child and adolescent psychiatry (in which, in case of urgency, a number of posts could in fact be created in the previous year). In mental handicap, expansion would depend on the number of vacant senior registrar posts filled prior to the year in question, and one would hope for an increase on the two posts allowed in 1982-83. Forensic psychiatry posts are likely to be related to medium secure units as these develop and, as noted above, psychotherapy posts will be automatically approved. Obviously what happens in practice will be dependent onfinancial resources as well as the supply of trained psychiatrists, and if the present pattern continues-on 31 March 1981 there was a backlog of 120 new psychiatric posts unfilled and 64 previously filled posts vacant for over a year-posts will have to be attractive to applicants. On the other hand, the 'Medical Fields of Recruitment' studies suggest that appointments committees are maintaining standards in that an analysis of posts advertised showed that over a third in adult and child and adolescent psychiatry were not filled in spite of an average of 2 to 4 applicants per post. Once again we have to say that training posts occupied by those who are "not able for one reason or another satisfactorily to complete specialist training to consultant level but wish to work part-time or full-time in the hospital service' might be released if their present occupants were successful in a personal application for employment in the associate specialist grade.

ASHLEY ROBIN

Ex-Chairman

Fiona CALdicotT

Chairman

\section{Defining one's terms}

\section{DEAR SIR}

In the September issue of the Bulletin (p. 168) Ezra the Scribe argued that as the training and experience of doctors are longer and broader than those of the other groups with whom they work they are, understandably, the group on whom responsibility for taking the major decisions falls. I do not wish to contribute to the discussion on responsibility, but my own training and experience lead me to question his notion that it is helpful to note the exact meaning of words when we are attempting to understand the concepts that those words embody. Perhaps it is more helpful to give consideration to history, context and the continuing evolution of language; or to put it more precisely, to understand exactly what we mean by 'exact'.

It was not difficult to determine that Ezra's search for exact meanings took him to the New English Dictionary (OUP) and reference to this work reveals that there are nine exact, and diverse, meanings listed for 'team' and twelve for 'discipline'. There must be many who would agree that for the purpose of understanding the developing concept of 'multi-disciplinary team' the most helpful of these exact meanings of 'team' would be 'a number of persons associated in some joint action' and of 'discipline' it would be 'a branch of instruction or education; a department of learning or knowledge', although I doubt if they would be acceptable to a nineteenth century farmer or a regimental sergeant major. We must be grateful that Ezra did not select as the exact meaning of discipline 'to scourge or flog by way of penance or the mortification of the flesh'.

With the dictionary beside me I decided to follow Ezra's own advice and noted that the exact meaning of 'scribe' is 'one who writes at another's dictation; chiefly with a contempuous notion, a party hack'. I write, dear Sir, to seek assurance that Ezra does not write at your dictation and that the party is not the Royal College of Psychiatrists.

21 Radnor Walk

London SW3

Frank TAIT

IThough one can dictate ('to put into words which are to be written down', OED) to Ezra there is little likelihood of its being transcribed. Ezra is a most independently-minded fellow!-Eds.]

\title{
Erratum
}

In Part II of 'In Conversation with Eliot Slater' (Bulletin, October) two amendments should be made. On page 179 , last paragraph, the actual words referred to were: 'For scientific purposes, the official scheme of classification just fails to be useless' (Annals of Eugenics (1935), 6, 177). On page 180 , column 2 , the text should have read: 'What happened eventually was a sad story'. 